\title{
Diffusion of Innovation in Competitive Markets A Study on the Global Smartphone Diffusion
}

\begin{abstract}
S. GÜNDÜÇ*
Ankara University Faculty of Engineering, Department of Computer Engineering Gölbaşı, Ankara, Turkey

(Received September 29, 2018; in final form January 22, 2019)

In this work, the aim is to study the diffusion of innovation of two competing products. The main focus has been to understand the effects of the competitive dynamic market on the diffusion of innovation. The global smartphone operating system sales are chosen as an example. The availability of the sales and the number of users data, as well as the predictions for the future number of users, make the smartphone diffusion a new laboratory to test the innovation of diffusion models for the competitive markets. In this work, the Bass model and its extensions which incorporate the competition between the brands are used. The diffusion of smartphones can be considered on two levels: the product level and the brand level. The diffusion of the smartphone as a category is studied by using the Bass equation (category-level diffusion). The diffusion of each competing operating system (iOS and Android) are considered as the competition of the brands, and it is studied in the context of competitive market models (product-level diffusion). It is shown that the effects of personal interactions play the dominant role in the diffusion process. Moreover, the volume of near future sales can be predicted by introducing appropriate dynamic market potential which helps to extrapolate the model results for the future.
\end{abstract}

DOI: 10.12693/APhysPolA.135.485

PACS/topics: diffusion of innovation, the Bass model, competitive markets, global smart phone market sales, artificial societies

\section{Introduction}

Diffusion of innovation studies has always been a significant part of the marketing strategies when a new product is introduced to the markets $[1,2]$. The changes in technologies and social behavior of the individuals affect the marketing strategies. Moreover, as the advanced technology products appear in the markets, more often similar products are introduced by different brands. Competition between the similar products of different brands can be both destructive or constructive. The existence of the competing products increases the word-of-mouth, which helps the growth of the market potential. Hence, gaining a better understanding of the diffusion of new products in competitive markets is gaining interest for both the scientists and the practising economists. Predictions on the conditions of success or failure of a new product in the competitive markets [2-4] may lead to the changes of policies, and even on the product specifications.

The first mathematical model of the diffusion of innovation in the literature is the Bass model [5]. The Bass model assumes a single product diffusion in a homogeneous and fully connected social network. The Bass model classifies the adoption characteristics of the individuals into two different groups. The first group is the individuals who decide to adopt the new product immediately after being informed. The external influences, such as advertisement plays the role of the driving force. This group of individuals is named as the innovators. The second group of individuals expects to see the advantages

*corresponding author; e-mail: gunduc@ankara.edu.tr of the product before adopting. Hence, in the adoption of the second group of individuals personal interactions with already adopted individuals or word-of-mouth is the crucial factor. The second group is called imitators since their decision depends on the number of adopted neighbors.

The Bass model contains three parameters. The probability of being affected by the external influences is called the innovation parameter $p$, while the number of imitators is controlled by the imitation parameter $q$. The potential market size is also another parameter which is considered as a constant in the original form of the Bass equation.

The Bass model well explains the diffusion of early technological innovations, such as household goods, agricultural innovations, automobiles, telephone, and postal banking systems [6]. In the early days of technological innovations, most of the time a brand could dominate a specific market, and the diffusion process could be studied in a restricted geographic area by the fixed size market potential assumption.

Recently, however, globalization, advancements in communication, and transportation technologies have changed the marketing considerations completely. Recent marketing observations indicate that almost always similar new products are introduced as competing brands $[7-9]$. The rules of diffusion are different when there is competition between the products. In the competitive market case, more than one type of internal influences exist. The potential adopters are not only influenced by the adopters of the product of interest, but also the adopters of the competitive products take a role in their decision-making process. These new type influences are called cross-brand or cross-product effects [9-12]. 
Moreover, the new products aim to reach the competitive global markets. Hence, the potential market size is dynamic [13, 14]. Even though there is no data-based evidence, Kim et al. [15-17] suggests that coexistence of competing products in the same market, indicates a potential for the products and helps the growth of the markets. Hence in the competitive market case, the dynamics of the potential market size need to be considered together with the dynamics of the adopters.

The existing literature on the studies of competitive markets varies from investigations of the diffusion of new software under piracy [11] to diffusion of minivans [9], or diffusion of mobile technologies and mobile phones $[18,19]$ to the diffusion of drugs of the same active components [20]. Modern communication systems and mobile phones have also gained considerable interest. As an advanced version of mobile communication technologies, the diffusion of smartphones and smartphone software are an exciting area of diffusion of innovation studies. The new smartphone operating systems, which are iOS and Android, have shown rapid success in the markets and increased the sales rate and market size. The success of these new operating systems has been the subject of various studies [21, 22]. Tseng et al. [21] have used a model, in which scenario analysis, expert opinion, Delphi, and widespread diffusion of innovation models are used in an integrated fashion to predict feature market shares of the smartphone operating systems. Wang et al. have also studied competition in the smartphone markets [22]. The Lotka-Volterra equation which is initially introduced for the competition of species is used for the short-term predictions for market shares of iOS and Android operating systems [22]. None of these studies have the power to predict the expansion of the potential market size. The relation between the timedependent potential market size and the market shares of competing products is the relatively less known area of research [20, 23-25].

The aim of the present work is threefold: the first one is to determine the values of external and internal influence parameters of the smartphone operating systems. The parameter values of previous technological innovations are already obtained and listed. Hence, a comparison of the innovation and imitation parameters of the smartphone operating system markets with the existing parameters will be illuminating. The second aim is to establish a functional form for the time dependence of the potential market size which has the power to predict future sales. Finally, the third one is the calculation of the growth of the potential market size due to the competition of the brands. In modern societies, markets are dynamic; market growth is a function of technological utility, economic, and social considerations as well as social prestige. Despite, the difficulty of writing an explicit form of the market potential regarding various social and economic parameters, parametric market potential can be used for the predictions. In this sense, the present work is a comparative study of the effects of fixed and the different forms of dynamic market potentials [20, 24].

In the computation of the diffusion of innovation of the smartphone operating systems, a two-stage process is adopted. As the first step, the category level diffusion parameters are obtained by using analytical and numerical solutions of the Bass equation for both timeindependent and dynamic market situations. As the second step, product-level internal, external, and competition parameters of the diffusion of innovation are obtained by fitting the data to the analytical and numerical solutions of coupled differential equations-Extended Bass equations. The parameters of the models are obtained by fitting the available smartphone sales and user data $[26,27]$ to the solutions of the Bass and generalized Bass equations.

The smartphone operating systems sales is a model for competitive markets, and it is a representative of modern diffusion processes in which global availability and fast information diffusion play an essential role [28]. For this reason, smartphone diffusion has some properties which are Sui Generis. First of all, it is straightforward to isolate a group of products from others which does not serve the same purpose. The smartphone operating systems, regardless of the brands, constitute the category. Secondly, only the products of the two brands dominate the whole market. Hence, the category has two competing products. Thirdly, the diffusion of both information and products are global, and shows very little difference in vast geographic distances. This property of the smartphone category enables the use of the aggregate models: the category-level diffusion of innovation can be studied by using the famous Bass equation [5], while alternative models can be used to study the competition between different products. In general, the models which aim to explain the dynamics of product-level diffusion are the generalizations of the Bass model. Krishnan et al. [9] introduced a model which connects category-level sales with product-level sales. The model introduced by Guseo $[29,30]$ has the same property. Muller et al. formulated the one which has fewer limitations compared to the others [8]. Models which establish the direct relation between the category-level and brand-level diffusion processes also exist [9-11, 30].

Many mobile phone producing companies have started the smartphones revolution. Starting from the first quarter of 2009, quarterly global sales [26] and global market shares [31] data for all smartphone operating systems are available. Immediately after the introduction of smartphone idea, two new operating systems which are introduced in 2007 and 2008, started to dominate global markets. Currently, only these two operating systems are dominating the global markets (in 2017 99.6\%) [32]. The number of actively used devices [32-34] were announced by producers of both operating systems. Apart, from the sales and shares data, forecasts on the number of smartphone users until 2020 are also available [26, 27]. 
Such information eases the modelling of data and enables to compare the prediction capabilities of different models.

In this work, the Bass equation has been used to calculate category level diffusion of smartphone operating systems. The Bass equation with constant market potential size is shown to capable of explaining the existing sales data but predicts that the sales will saturate after 2017. Different time-dependent potential market models eliminate this shortcoming, and for the category level sales, the Bass model predictions and predictions based on social and economic considerations [27] matches well. Brand level sales predictions require the use of generalized Bass model type aggregate models. Three such models are considered by using both constant and timedependent potential market size $[8,9,30]$. The existing data provide an excellent platform to test various diffusion of innovation models under competition. The results have shown that the smartphone markets are still in their growth phase.

The following section is devoted to the discussions on the existing competitive market models. In the third section, the smartphone operating system sales data is considered. In this section, the diffusion of innovation parameters are also obtained by using the smartphone sales data, future predictions, and a mathematical model of the competing markets. In the last section, discussions of the results and conclusions are presented.

\section{Innovation of diffusion models}

The Bass equation [5] defines the diffusion of innovation in terms of three parameters. The adoption rate at time $t$ is given by the equation

$$
\frac{\mathrm{d} N(t)}{\mathrm{d} t}=\left(p+q \frac{N(t)}{M(t)}\right)[M(t)-N(t)],
$$

where $p, q$, and $M$ are the external (innovation) and internal (imitation) influence, and the market potential size parameters, respectively. $N(t)$ is the number of adopters at a given time $t$. In the original Bass equation (Eq. (1)), the relation between the already adopted and the potential adopters, are represented by a linear relation and the market potential size $M(t)=M$ is fixed. Hence, market potential size is an upper bound of the diffusion process.

If the same market is shared by more than one product, the competition changes the dynamics of the diffusion. Adopters of competing products affect all potential adopters. The existence of competing products requires the introduction of cross-product influences. The influence of the adapters on the potential adopters of competitive products may be positive or negative. Hence, competition may even increase the sales of competing products. The total sales of all brands constitute the category; thus the market gain of one product does not directly mean a market loss of the other.

The simplest model of competition is introduced by Krishnan et al. [9]. In this model category-level as well as the product-level diffusion equations are simple Bass equations. The coupling between the simple Bass equations are satisfied with the restriction that the product level external, $p_{i}$, and internal, $q_{i}$, parameters add up to the category level external, $p_{c}$, and internal, $q_{c}$, parameters. The Krishnan-Bass-Kumar model (KBKM) assumes that the potential adopters, $M(t)-N(t)$, and market potential size $M(t)$, are common for both category and product level equations.

$$
\begin{aligned}
\frac{\mathrm{d} N(t)}{\mathrm{d} t} & =\left(p_{c}+q_{c} \frac{N(t)}{M(t)}\right)[M(t)-N(t)], \\
\frac{\mathrm{d} N_{i}(t)}{\mathrm{d} t} & =\left(p_{i}+q_{i} \frac{N(t)}{M(t)}\right)[M(t)-N(t)] \\
\text { where } i & =1, \ldots, k .
\end{aligned}
$$

Here, $k$ is the number of products which are sharing the same market and $p_{c}, p_{i}$ and $q_{c}, q_{i}$ are the category-level and product level external and internal influence parameters, respectively.

The constraint relations

$$
p_{c}=\sum_{i}^{k} p_{i}, \quad q_{c}=\sum_{i}^{k} q_{i}, \quad N(t)=\sum_{i}^{k} N_{i}(t)
$$

ensures the correct inter- and cross-product level influences. Here, $N(t)$ is the total number of adopters which is the sum of the adopters of all competing products, $N_{i}(t)$ at the market.

The main disadvantage of the Bass-Kumar model [9] is that the relative effects of the influences on the adopters of the competing products are not explicitly expressed in the model.

Guseo and Guidolin [29] proposed a model for the market potential growth. In the same work, the Bass model is extended by an additional correction term to account a self-reinforcing effect of the dynamic markets (Guseo and Guidolin model, GGM):

$$
\begin{aligned}
& \frac{\mathrm{d} N(t)}{\mathrm{d} t}=\left(p+q \frac{N(t)}{M(t)}\right)(M(t)-N(t))+N(t) \frac{M^{\prime}(t)}{M(t)}, \\
& p, q>0 ; t \geq 0,
\end{aligned}
$$

where $p$ and $q$ are external and internal influences, $N(t)$ and $M(t)$ are the number of adopters and dynamic market size at time $t$. When the market potential is constant, Eq. (4) becomes the Bass equation.

Later, Guseo and Mortarino have extended Eq. (4) to describe the diffusion of two competing products [20,30]. Two equivalent forms of coupled differential equations and their analytical solution is presented in Ref. [30]. The model proposed by Guseo and Mortarino will be named GMK hereafter.

One of the equivalent models of competitive markets is given as

$$
\begin{aligned}
& \frac{\mathrm{d} N_{1}(t)}{\mathrm{d} t}=\left(p_{1}+q_{1} \frac{N(t)}{M(t)}+\delta \frac{N_{1}(t)}{M(t)}\right) \\
& \times(M(t)-N(t))+N_{1}(t) \frac{M^{\prime}(t)}{M(t)},
\end{aligned}
$$




$$
\begin{aligned}
& \frac{\mathrm{d} N_{2}(t)}{\mathrm{d} t}=\left(p_{2}+\left(q_{2}-\delta\right) \frac{N(t)}{M(t)}+\delta \frac{N_{2}(t)}{M(t)}\right) \\
& \times(M(t)-N(t))+N_{2}(t) \frac{M^{\prime}(t)}{M(t)},
\end{aligned}
$$

where the parameters $p_{1}, P_{2}, q_{1}, q_{2}$, and $\delta$ controls the diffusion and the competition between the products. One of the most intriguing features of Eq. (5) is that the addition of two equations gives the category level diffusion equation of the products. Hence, GMM is also equivalent to KBKM.

Interactions between the adopters and potential adopters of competing products are also studied in alternative models. Majority of these models are generalizations of the Bass model. Libai formulates the one which has fewer limitations compared with the others

$$
\begin{aligned}
& \frac{\mathrm{d} N_{i}(t)}{\mathrm{d} t}=\left(p_{i}+\sum_{j} q_{i, j} \frac{N_{j}(t)}{M}\right)(M-N(t)), \\
& i, j=1, \ldots, k,
\end{aligned}
$$

where $k$ is the number of competing products, $p_{i}$, and $q_{i, j}$ are the innovation, and imitation parameters of the $i$-th product. The model introduced by Libai et al. [8] hereafter will be called LMPM. In this model for each product, there exist more than one imitation parameters. $q_{i, i}, i, j=1, \ldots, k$ and $q_{i, j}, i, j=1, \ldots, k ; j \neq i$ are the inter-product and cross-product imitation parameters, respectively. The cross-product parameters, $q_{i, j}$, are the measure of the degree to which adoption of the $i$-th product is affected by the adopters of the $j$-th competing product [24].

The dynamic market growth is an interesting topic. After the introduction of the product, new demand may be created and time-dependent increase in the market size can be observed. Various form of dynamic market growth is introduced in the literature.

The simplest market growth forms are the exponential time evolution of the market size $M(t)$ [35-37]:

$$
M(t)=M(0) \exp (\delta t), \text { where } \delta>0 .
$$

The Bass equation with exponentialy growing market potential is also solved analytically [35].

Another form is introduced by Namwoon [38]:

$$
M(t)=M(0)(1-\exp (-\delta C(t))),
$$

where $M_{0}$ is the maximum potential market size, $C(t)$ is the number of competitors at time $t$.

In the smartphone operating system sales case, there are only two competitors in the long term, the form used by Namwoon et al. is simplified to parametrize the time dependence of the market growth, in this work.

$$
M(t)=M(0)(1-\exp (-\delta t)) \quad \delta>0 .
$$

Here, $\delta$ is the time constant of the market growth.

Recently, Guseo and Guidolin have introduced a new form of market potential growth [29]. This form has obtained diffusion model based on cellular automata dynamics. The introduced form of the growth is basically the same as the Bass model: growth of the awareness of the potential adopters grows the market potential. The obtained form

$$
M(t)=M_{0} \sqrt{Z(t)},
$$

where

$$
Z(t)=\frac{1-\exp \left(-\left(p_{c}+q_{c}\right) t\right)}{1+q_{c} / p_{c} \exp \left(-\left(p_{c}+q_{c}\right) t\right)}
$$

is used in studies of diffusion of competing drugs [30].

Equations (2)-(6) are solved under the assumptions that the potential market size is constant and the potential market size is growing in time. The consequences of having constant market potential size and time dependent growth in the market potential size are very important, which directly affects the prediction power of the models.

In the following section, the models will be applied to smartphone operating system sales data. The relative importance of diffusion parameters, effects of the competition, and the diffusion processes will be discussed.

\section{Results and discussions}

In this section, the prediction power of different diffusion of innovation models and the effect of market growth will be tested by obtaining the parameter values from the data. The available data are in two groups: the first group consists of quarterly sales of competing brands which are obtained from statistics [26], the second set is the number of actively used devices starting from 2016 which also include predictions until 2020 [27].

Figure 1 shows that the global sales increase at every quarter while the contributions of the brands other than iOS and Android are rapidly decreasing.

The cumulative smartphone sales data which is necessary for comparison with the model prediction can be obtained from quarterly sales as

$$
S_{i}^{a}=\sum_{j=1}^{i} s_{j}^{a},
$$

where $s_{j}^{a}$ and $S_{i}^{a}$ represent sales and cumulative sales of brand $a$ at the $j$-th time slice, respectively. The global cumulative sales (Eq. (12)) obtained from the data, Fig. 1, are much higher than the number of existing smartphones [27]. This discrepancy can be explained by considering relatively short active lifespan of smartphones. To obtain sensible results, active product lifespan must be taken into consideration. The difference can be seen in Fig. 2.

The proposed empirical model assumes that every year a fraction of the existing devices are becoming unusable. Hence, this fraction must be reduced from the cumulative sum

$$
S_{i+1}^{a}=(1-f(i)) S_{i}^{a}+N_{i+1}^{a},
$$

where $f(i)$ is a time dependent factor representing the fraction of the devices that are dropped from the sum. For simplicity, the form of the function is assumed linear 


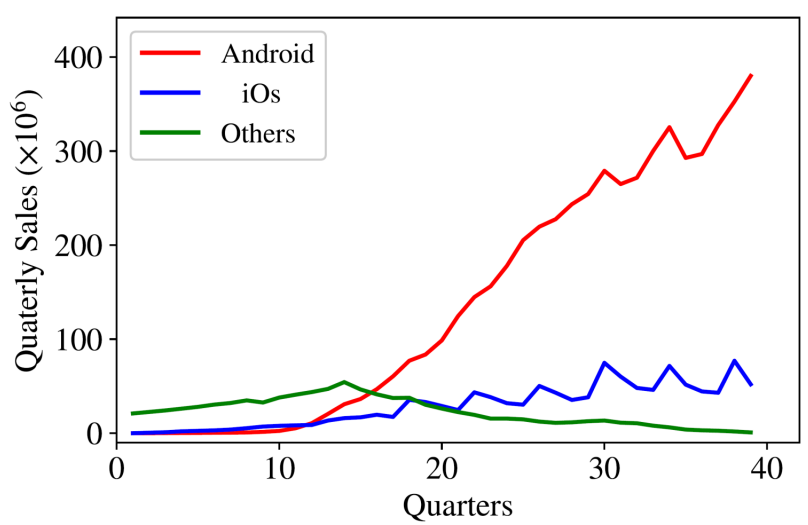

Fig. 1. Global sales of smartphones operating systems starting from the first quarter of 2009.

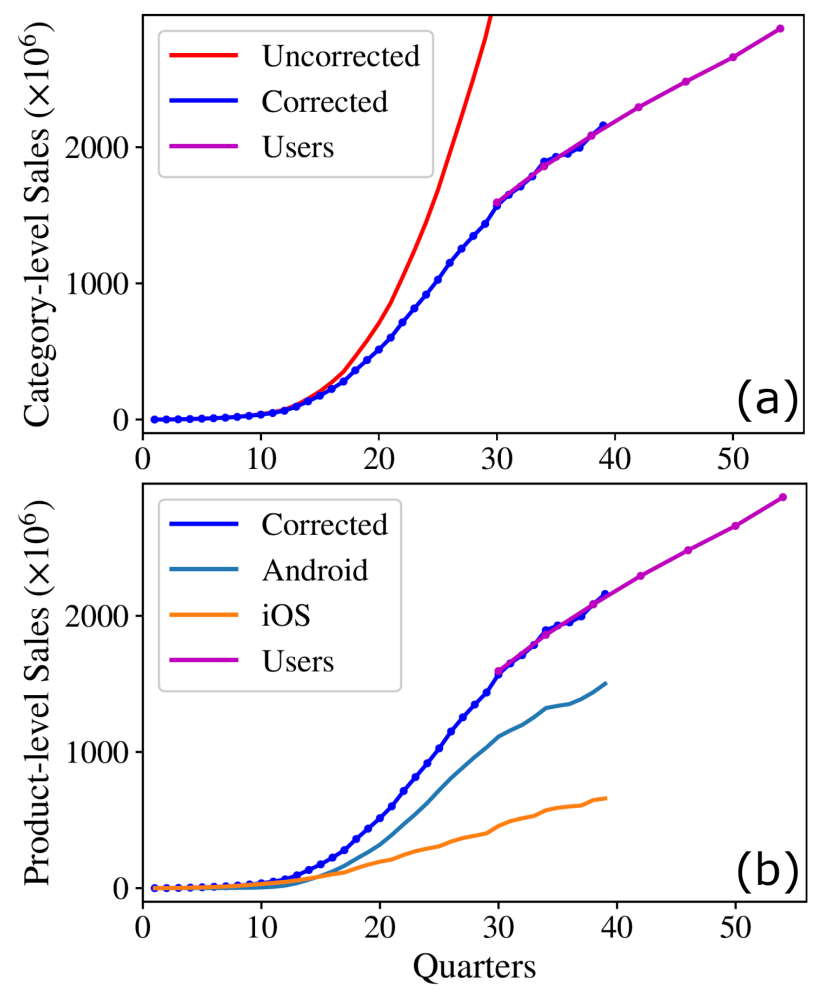

Fig. 2. Global cumulative sales of smartphones with iOS and Android operating systems starting from the first quarter of 2009: (a) Cumulative category-level sales, (b) cumulative product-level sales.

$$
f(t)=f_{0}+f_{1} t .
$$

The parameters of the reduction function (Eq. (14)) are obtained by fitting the processed cumulative sales data and the actual users data $[27,32,33]$. The best matching form (Eqs. (13) and (14)) is obtained by an empirical fitting process. The obtained coefficients $f_{i}^{a}$ are given in Table I.

The category-level cumulative numbers are calculated for each quarter by adding product-level cumulative numbers. Figure 2a shows the cumulative numbers without and with the correction term. The number of active smartphone users are also presented for comparison.
TABLE I

Parameters of empirical correction function.

\begin{tabular}{l|c|c}
\hline \hline & $f_{0}^{(2)}$ & $f_{1}^{(2)}$ \\
\hline Android & 0.110 & 0.003 \\
iOS & 0.025 & 0.001
\end{tabular}

\subsection{Model applications}

The diffusion of smartphones is studied at two levels: category and brand levels. First, the category level diffusion is discussed. The Bass Eq. (1) and the model introduced by Guseo and Guidolin [29] with different dynamic market potentials are employed to study the internal and external effects on global smartphone diffusion. Second, three different but related competitive market models with dynamic market potentials are tested on the smartphone data. The global quarterly sales data indicate no saturation (Fig. 1) which shows that the potential market is increasing as the diffusion of this new technology spreads out. The discussions on the form and the parameter values of competition together with the dynamic market growth will be the focal point of this section.

\subsubsection{Category level diffusion of innovation}

Figure 3 shows that the Bass model fits reasonably well to the existing data. The shortcoming of the Bass equation with constant market potential (Fig. 3a) is its prediction of saturation in sales. The saturation is seen immediately after the date, where the data points end. Hence, there is a serious discrepancy between the Bass model predictions and the forecasts on the feature sales potential. To overcome this shortcoming of the Bass model, three different dynamic market potential forms are tested (Fig. 3b-d). The contribution of the self-reinforcing effect of the dynamic markets [29] are also tested. For all dynamic market models, conclusions for both Bass and Bass with additional correction term (GGM) are the same.
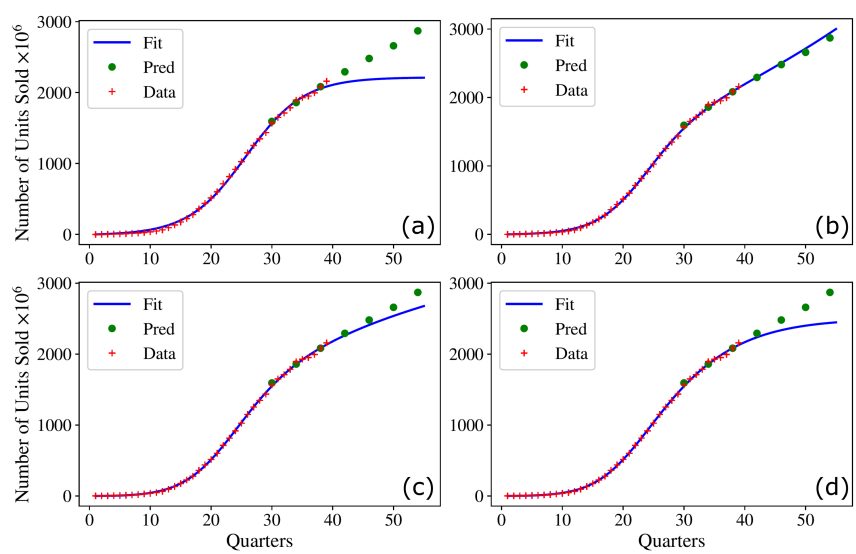

Fig. 3. Cumulative smartphone sales modelled by using Bass equation with four different market definitions: (a) constant market size, (b) model I, (c) model II, (d) model III. 
The resemblance between the existent data and the fits are very good for all four cases. The fit parameters, standard deviations, and goodness of the fit $\left(R^{2}\right)$ values for all four models of market potential are presented in Table II. In the table, $M_{I}, M_{I I}$, and $M_{I I I}$ indicate dynamic market models defined by Eqs. (7), (9), and (10), respectively. As far as the cumulative sales data until the year 2017 are concerned, constant and time-dependent growing market potential models fits are very satisfactory. Two main criteria, standard deviation and $R^{2}$ comparisons indicate that the dynamic market potential case is slightly better.

TABLE II

Fit parameters of Bass model with four different dynamic market potential definitions.

\begin{tabular}{|c|c|c|c|c|}
\hline & $M=\mathrm{const}$ & Model I $\left(M_{\mathrm{I}}\right)$ & Model II $\left(M_{\mathrm{II}}\right)$ & Model III $\left(M_{\mathrm{III}}\right)$ \\
\hline$p$ & $\begin{array}{c}1.1543 \times 10^{-3} \\
\pm 8.3080 \times 10^{-5}\end{array}$ & $\begin{array}{c}1.3251 \times 10^{-3} \\
\pm 8.1876 \times 10^{-5}\end{array}$ & $\begin{array}{c}2.1652 \times 10^{-3} \\
\pm 1.2786 \times 10^{-4}\end{array}$ & $\begin{array}{c}2.7556 \times 10^{-3} \\
\pm 9.2427 \times 10^{-4}\end{array}$ \\
\hline$q$ & $\begin{array}{c}2.0899 \times 10^{-1} \\
\pm 4.5457 \times 10^{-3}\end{array}$ & $\begin{array}{c}2.3685 \times 10^{-1} \\
\pm 5.7023 \times 10^{-3}\end{array}$ & $\begin{array}{c}2.1568 \times 10^{-1} \\
\pm 6.1231 \times 10^{-3}\end{array}$ & $\begin{array}{c}2.2184 \times 10^{-1} \\
\pm 9.6020 \times 10^{-3}\end{array}$ \\
\hline$M_{0}$ & $\begin{array}{c}2.2144 \times 10^{3} \\
\pm 2.1684 \times 10^{1}\end{array}$ & $\begin{array}{c}1.0099 \times 10^{3} \\
\pm 7.4212 \times 10^{1}\end{array}$ & $\begin{array}{c}3.9004 \times 10^{3} \\
\pm 6.2595 \times 10^{2}\end{array}$ & $\begin{array}{c}2.5013 \times 10^{3} \\
\pm 1.7755 \times 10^{2}\end{array}$ \\
\hline$\delta$ & - & $\begin{array}{c}1.9810 \times 10^{-2} \\
\pm 1.8909 \times 10^{-3}\end{array}$ & $\begin{array}{c}2.1116 \times 10^{-2} \\
\pm 5.3071 \times 10^{-3}\end{array}$ & $\begin{array}{l}- \\
- \\
\end{array}$ \\
\hline$p_{M}$ & - & - & - & $\begin{array}{c}2.6312 \times 10^{-3} \\
\pm 2.4338 \times 10^{-3}\end{array}$ \\
\hline$q_{M}$ & $\begin{array}{l}- \\
-\end{array}$ & $\begin{array}{l}- \\
-\end{array}$ & $\begin{array}{l}- \\
-\end{array}$ & $\begin{array}{c}1.2489 \times 10^{-1} \\
\pm 4.6963 \times 10^{-2}\end{array}$ \\
\hline $\begin{array}{c}\sigma \\
R^{2}\end{array}$ & $\begin{array}{c}25.5307 \\
0.9989\end{array}$ & $\begin{array}{l}15.1516 \\
0.9996\end{array}$ & $\begin{array}{c}13.74302 \\
0.9997\end{array}$ & $\begin{array}{l}14.1204 \\
0.9997\end{array}$ \\
\hline
\end{tabular}

One can argue that dynamic market potential definitions increase the number of parameters of the model. Therefore, the existence of extra parameters may increase the flexibility of the fit function and may result in smaller standard error and better $R^{2}$ value. For all three different dynamic market models (Eqs. (7), (9) and (10)), F-tests are performed (Table III). The F-test values give clear indication that the Bass model with the dynamic market potential models fit better than the constant market potential.

From the marketing point of view, steep increase in the quarterly sales is an indication of growing market size. Moreover, global smartphone users data and predictions on the future number of users indicate that the cumulative sales are far from a saturation situation [27].

The main test of the dynamic market models is their prediction power. All three dynamic models are put to test for predicting future sales. The fits to the existing quarterly sales data extrapolated until the year 2020. The extrapolations are compared with the existing prediction data. Figure 3 shows the fit and extrapolation regions of all market potentials. All of the dynamic market models exhibit improvements over the constant market case. Nevertheless, first two models (model I, Fig. 3b and model II, Fig. 3c) are better for predicting near future sales. As a conclusion of this subsection, it can be said that the best agreement between the predictions and

\section{TABLE III}

F-test results: Model comparisons between the constant and dynamic market potential solutions of the Bass model.

\begin{tabular}{c|c|c|c}
\hline \hline$F_{M_{I}}$ & $F_{M_{I I}}$ & $F_{M_{I I I}}$ & $F_{M_{I V}}$ \\
\hline 44.46 & 89.24 & 41.84 & 67.21
\end{tabular}

the solution of the Bass equation can be reached when the potential market exhibits exponential growth with time $\left(M_{I}\right)$.

\subsubsection{Product level diffusion of innovation}

To compare the effects of different dynamic market potentials under competition, four different market potentials are presented in the context of three diffusions of innovation models. In this section, three models which are introduced by Kirshnan-Bass-Kumar (KBKM) [9], Guseo-Mortarino (GMM) [30] and Libai-Muller-Peres (LMPM) [8], are compared. The model parameters together with the parameters of dynamic market potentials require an extra care for the fitting procedure. Since, the dimensions of the parameter space is high, to avoid the local minima, a random search is performed to choose the lowest standard deviation. The lowest standard deviation value is chosen from 500 minimization runs with random initial parameter sets used to determine the lowest standard deviation value. The parameter set which minimizes 

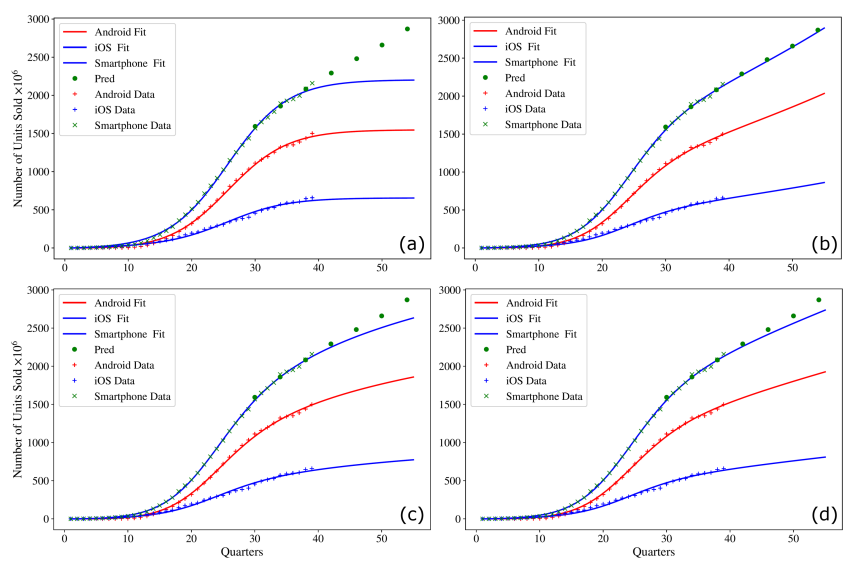

Fig. 4. Cumulative smartphone sales, modelled by using Krishnan-Bass-Kumar model [9], with the assumptions: (a) the potential market size is constant, (b)-(d) dynamic market potential cases, $M_{I}, M_{I I}$, and $M_{I I I}$.
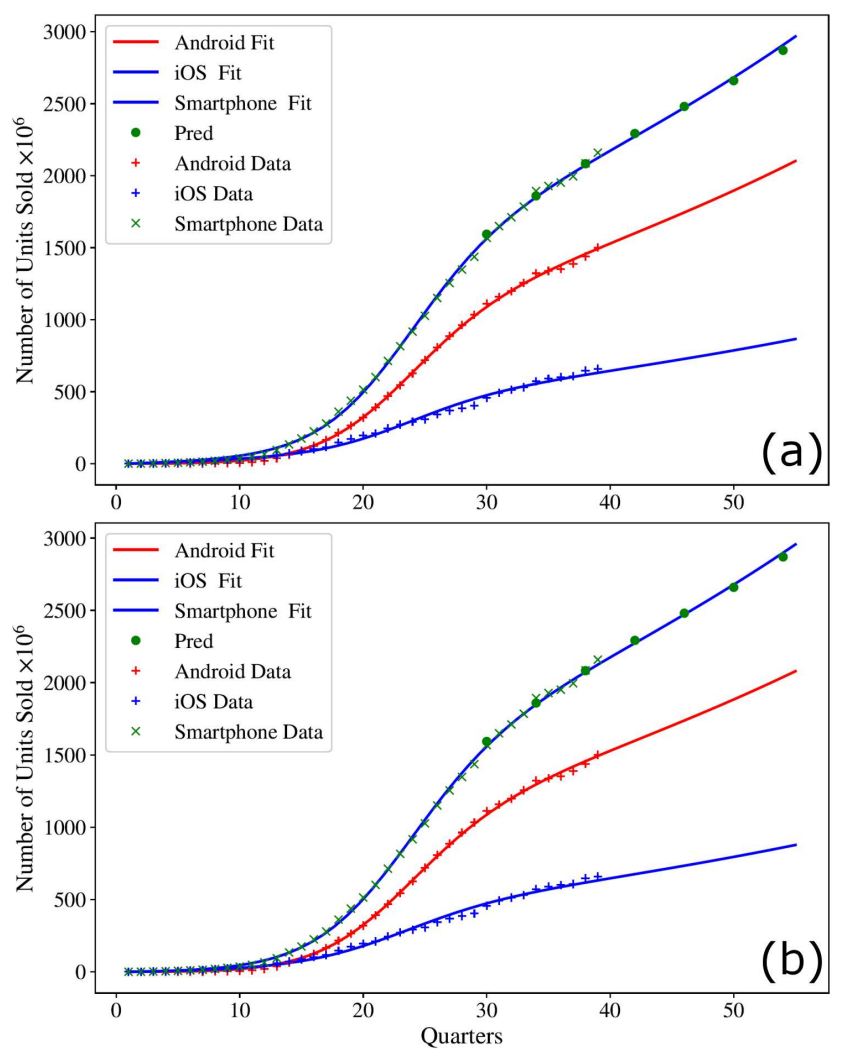

Fig. 5. Predictions of cumulative smart phone sales (under exponentially growing market assumption): (a) L model with 6 parameters, LMPM, (b) GMM with 5 parameters.

the standard deviation is presented as the parameter set in the results. The number of available data points are sufficient for the estimation of the parameters. Nevertheless, sophisticated statistical analysis require more data points for the given number of parameters. Despite all the efforts, the error estimation using convolution matrix or bootstrap methods cannot give satisfactory results. Hence, parameter error estimates are not presented in this subsection.

Solutions of coupled differential equations, for all three models are obtained by using SciPy odeint packet if no analytical form exists [30]. The models of diffusion of innovation (KBKM, GMM, LMPM) are very successful fitting the existing quarterly cumulative sales data. The discrepancy starts with the prediction of the future sales. To discuss the effects of the dynamic market potentials, all three diffusion models are solved by using alternative market potential forms. Results are very similar, and particularly in the graphical representation of the data and fits are almost indistinguishable up to the end of quarterly sales data, the first quarter of 2017.

As an illustrative example, the results of the KirshnanBass-Kumar model will be presented in detail, and the other two models, GMM and LMPM, will be added to discussions when additional arguments are presented. Figure 4 shows the solutions of the KBKM with constant and dynamic market potential definitions. Figure $4 \mathrm{a}$ shows the characteristic S-shape of the diffusion of the innovation curve. With constant market potential approximation, the maximum size of the market is determined as $2.2077 \times 10^{+9}$ customers. This is approximately one-third of the world population and this number is the estimated/observed number of smartphone users in 2017 [32-34]. Despite such a wide usage, still smartphone market is growing globally. Hence, market potential forecasts does not agree with obtained saturation level. Second sub-figure (Fig. 4b) shows the results obtained by using exponentially growing market potential. This model is unrealistic in the long run, since no market can grow indefinitely. Nevertheless, it is in very good agreement with the existing data and the short term predictions on the number of future users. The fit to the cumulative quarterly sales data agrees well, with $\chi^{2}=37.6642$ and $R^{2}=0.9979$. Moreover, when the fit is extended until the year 2020, the agreement with the predictions are remarkably good $\left(\chi^{2}=12.2141\right.$ and $\left.R^{2}=0.9992\right)$. The other two dynamic market potential models also exhibit agreement with the existing quarterly sales data (Fig. 5a and b) but both predict saturation immediately after 2017. Hence, only exponentially growing market approximation give correct information for the short-term sales prediction.

Table IV shows parameter values, standard deviations, and r-squared values of the fits for all four different market potential forms. The KBKM fits indicate that the leading effects on the category level sales are the internal influences. As a consistency check, the values obtained for the category level parameters, $p_{c}$, and $q_{c}$ (in Eq. (2)) are also in accord with the parameter values obtained from the category level sales studies (Table II). The product level sales are governed by two sets of parameters which add up to the category level parameter values. Table IV indicates that for KBKM, the external influences play no role in the sales of the first product 
(Android OS). On the other hand, a considerable number of consumers buy the second product (iOS) as soon as it is announced. The effects of the internal influences are larger for the first product (Android) than the second product (for constant market size case, $p_{1} \sim p_{c}$ ). Effects of the internal influences are larger for the first product (Android) than the second product, which indicate that hands-on-experience and personal interactions play crucial role in the spread of the first product. Even for the dynamic market case this conclusion does not change.

TABLE IV

Bass-Kumar model parameters for four different market potential forms.

\begin{tabular}{c|c|c|c|c}
\hline \hline Parameter & $M 0$ & $M 4$ & $M 1$ & $M G$ \\
\hline$p_{c}$ & $1.1308 \times 10^{-3}$ & $1.1715 \times 10^{-3}$ & $2.0493 \times 10^{-3}$ & $2.2960 \times 10^{-3}$ \\
$q_{c}$ & $2.1047 \times 10^{-1}$ & $2.5453 \times 10^{-1}$ & $2.1569 \times 10^{-1}$ & $2.1766 \times 10^{-1}$ \\
$p_{1}$ & 0 & 0 & 0 & 0 \\
$q_{1}$ & $1.5213 \times 10^{-1}$ & $1.8161 \times 10^{-1}$ & $1.5825 \times 10^{-1}$ & $1.6025 \times 10^{-1}$ \\
$M_{0}$ & $2.2076 \times 10^{3}$ & $1.1562 \times 10^{3}$ & $3.4796 \times 10^{3}$ & $2.5412 \times 10^{3}$ \\
$\delta_{M}$ & - & $1.8163 \times 10^{-2}$ & $2.5320 \times 10^{-2}$ & - \\
$p_{M}$ & - & - & - & $4.8378 \times 10^{-3}$ \\
$q_{M}$ & - & - & - & $9.9715 \times 10^{-2}$ \\
$\sigma$ & 41.4162 & 37.6642 & 36.6009 & 37.6643 \\
$R^{2}$ & 0.9974 & 0.9979 & 0.9980 & 0.9979
\end{tabular}

Competition between the rival products is not openly pronounced in the Kirshnan-Bass-Kumar model. The other two models have some extra parameters designated to measure the contribution of the competition. The LMPM model contains two external parameters, $p_{1}$ and $p_{2}$ and four internal parameters, $q_{1}, q_{2}, c_{1}$, and $c_{2}$. The internal parameters, $c_{1}$ and $c_{1}$ are related with the cross-brand influences. GMM has two external parameters, $p_{1}$ and $p_{2}$, which have exactly the same as KBKM and LMPM, three parameters, $q_{1}, q_{2}$, and $\delta$, are related with the internal effects which have slightly different meaning. The parameter $\delta$ is employed to represent the cross-brand effects. Comparing internal parameters of LMPM and GMM, the correspondence can be given as, $c_{1}^{L M P M}=q_{1}^{G M M}, c_{2}^{L M P M}=q_{2}^{G M M}-\delta$. Tables $\mathrm{V}$ and VI show the parameter values obtained using dynamic market growth with exponential time dependence $M_{I}$. Both models predict positive cross-brand contribution. Both operating systems benefit from the existence of the other operating system.

For both models, the fitted parameters of the dynamic market potential is given in Table VII.

Figure 5 shows category level diffusion modelled by using LMPM and GMM models. Both models accompany exponentially growing dynamic market potential. The chi-square and r-square values give the quality of the fit for LMPM, $\chi^{2}=39.2933, R_{1}^{2}=0.9979$, and for GMM $\chi^{2}=37.0053, R_{1}^{2}=0.9980$.

Figure 5 also shows that for both models exponential growing market model successfully predict the values obtained [27]. The extrapolation of the fit matches the prediction data very well. For LMPM, $\chi_{2}=22.3559$, $R_{2}^{2}=0.9975$ and for GMM, $\chi_{2}=17.6432, R_{2}^{2}=0.9983$.
LMPM parameters.

TABLE V

\begin{tabular}{c|c}
\hline \hline Parameter & Value \\
\hline$p_{1}$ & 0.0000 \\
$q_{1}$ & $2.6573 \times 10^{-1}$ \\
$p_{2}$ & $2.8145 \times 10^{-3}$ \\
$q_{2}$ & $1.4201 \times 10^{-4}$ \\
$c_{1}$ & $5.9847 \times 10^{-2}$ \\
$c_{2}$ & $1.0758 \times 10^{-1}$
\end{tabular}

GMM parameters.

TABLE VI

\begin{tabular}{c|c}
\hline \hline Parameter & Value \\
\hline$p_{1}$ & 0.0000 \\
$q_{1}$ & $1.0721 \times 10^{-1}$ \\
$p_{2}$ & $1.1247 \times 10^{-3}$ \\
$q_{2}$ & $1.4072 \times 10^{-1}$ \\
$\delta$ & $1.1136 \times 10^{-1}$
\end{tabular}

TABLE VII

Exponentially growing dynamic market potential parameters for LMPM and GMM.

\begin{tabular}{c|c|c}
\hline \hline & $M_{0}$ & $\delta_{M}$ \\
\hline LMPM & $1.0607 \times 10^{3}$ & $2.0029 \times 10^{-2}$ \\
GMM & $1.0076 \times 10^{3}$ & $1.9565 \times 10^{-2}$
\end{tabular}

\section{Conclusions}

The Bass model has a great success in explaining diffusion mechanisms of an extensive range of products. Despite its success, the Bass model cannot accommodate 
economic and social parameters which have a direct influence on the sales and market success of a new product. Attempts to extend the Bass model to consider social and economic parameters complicate the model, since the parameters such as price, utility, social prestige, competition-driven popularity have different weights and importance for different product categories. Extra complications restrict the prediction power of the model. The most notable effect of all these parameters is to increase or decrease the demand. Hence, the above mentioned economic and social parameters are the driving forces of the dynamics of market growth.

In this work, answers to three main conceptual questions are searched: (a) what are the relative importance of the external and internal influences of a high technological innovation?, (b) how one can predict the sales for the near future?, (c) what is the effect of the competition in smartphone diffusion of innovation? Diffusion of innovation model with constant market size may give reasonable answers to these questions if the model is applied to the existing data, after the product diffusion reached its saturation level. The main concern, if one can predict future sales by using Bass or extended Bass model must accommodate the information on the dynamics of market growth.

In this work, the diffusion of smartphones operating systems is studied by using models presented in Sect. 2 and the existing data. Diffusion of smartphones has an exceptional place in the diffusion of innovation studies [28]. First of all, there is no other technological innovation which substitutes so many different technological appliances. Secondly, the social impact of smartphone technology has no match. Thirdly, smartphone technology is a living technology - frequent introduction of new versions with advanced features wet the appetite of the potential adopters and boost the sales. Two operating systems, namely Android and iOS dominate the smartphone markets. Hence, smartphone operating systems provide a laboratory for competitive market studies.

The relative importance of the external and internal influences are studied by fitting the quarterly sales data with the solutions of different diffusion of innovation models. It is observed that both in the category and the product level, external influences are quite insignificant. Instead, internal influences are the leading effects in both category and the brand level sales. This observation is related to the product itself. Smartphones create a new type of social interaction, namely social media, which enabled individuals to share experiences and opinions. The social media created a different type of trusted information source, which is the most influential factor in decision-making processes.

The near future sales prediction can be made by introducing a dynamic market potential. Any market potential with saturation can be used if the limits of the market size are known. On the other hand, for short term predictions, market models with time-dependent growth fits better (Fig. 4).
High competition helps the growth of the market by increasing the awareness of the potential adopters. It is seen from the inter-product competition terms (Tables $\mathrm{V}$ and VI) that the existence of positive competitors contribute positively to the sales of both brands.

\section{References}

[1] E.M. Rogers, Diffusion of Innovations, Free Press of Glencoe, New York 1962.

[2] E.M. Rogers, Diffusion of Innovations, 5th ed., Free Press, New York 2003.

[3] V. Mahajan, E. Muller, J. Wind, New Product Diffusion Models, Kluwer Academic, New York 2000.

[4] N. Meade, T. Islam, Int. J. Forecast. 22, 519 (2006).

[5] F.M. Bass, Manage. Sci. 15, 215 (1969).

[6] T. Hagerstrand, Innovation Diffusion as a Spatial Process, Univ. Chicago Press, 1968.

[7] S. Savin, C. Terwiesch, Operat. Res. 53, 26 (2005).

[8] B. Libai, E. Muller, R. Peres, J. Market. 73, 19 (2009).

[9] T.V. Krishnan, F.M. Bass, V. Kumar, J. Market. Res. 37, 269 (2000).

[10] M. Hahn, S. Park, L. Krishnamurti, A.A. Zoltners, Market. Sci. 13, 224 (1994).

[11] M. Givon, V. Mahajan, E. Muller, J. Market. 59, 29 (1995).

[12] C.E. Laciana, G. Gual, D. Kalmus, N. Oteiza-Aguirre, S.L. Rovere, Physica A 413, 104 (2014).

[13] V. Mahajan, R.A. Peterson, Manage. Sci. 24, 1589 (1978).

[14] V. Mahajan, E. Muller, F.M. Bass, J. Market. 54, 1 (1990).

[15] N. Kim, E. Bridges, R.K. Srivastava, Int. J. Res. Market. 16, 95 (1999).

[16] C. Van den Bulte, S. Stremersch, Market. Sci. 23 , 530 (2004).

[17] R.J. Kauffman, A.A. Techatassanasoontorn, Inform. Technol. Manage. 6, 253 (2005).

[18] H. Gruber, F. Verboven, Europ. Econom. Rev. 45 577 (2001).

[19] A. Scharl, A. Dickinger, J. Murphy, Electron. Commerce Res. Appl. 4, 159 (2005).

[20] R. Guseo, C. Mortarino, IMA J. Manag. Math. 25, 287 (2014).

[21] F.M. Tseng, Y.L. Liu, H.H. Wu, J. Eng. Technol. Manage. 32, 40 (2014).

[22] H.T. Wang, T.C. Wang, Technol. Anal. Strateg. Manage. 29, 414, (2016).

[23] P. Parker, H. Gatignon, Int. J. Res. Market. 11, 17 (1994).

[24] R. Peres, E. Muller, V. Mahajan, Int. J. Res. Market. 27, 91 (2010).

[25] R. Guseo, C. Mortarino, Europ. J. Oper. Res. 216, 658 (2012).

[26] Quaterly sales data: All operating systems are included, data starts from the first quarter of the year 2009 . 
[27] Actual data and forecasts on the number of smartphone user between 2014 and 2020.

[28] M. DeGusta, Are Smart Phones Spreading Faster than Any Technology in Human History? MIT Technol. Rev. (Busin. Rep.) May 9, 2012.

[29] R. Guseo, M. Guidolin, Technol. Forecast. Soc. Change 76, 806 (2009).

[30] R. Guseo, C. Mortarino, Ann. Appl. Statist. 9, 2073 (2015).

[31] Quaterly shares data: All operating systems are included, data starts from the first quarter of the year 2009.

[32] The market share of smartphones run Android or iOS is 99.6 .
[33] The actively used iOS operating system based devices exceed 700 milion worldwide at the beginning of 2017 .

[34] There exists more than 2 bilion actively used Android operating system based devices worldwide at the beginning of 2017.

[35] M.N. Sharif, K. Ramanathan, Technol. Forecast. Soc. Change 20, 63 (1981).

[36] P. Meyer, J.H. Ausubel, Technol. Forecast. Soc. Change 61, 209 (1999).

[37] F. Centrone, A. Goia, E. Salinelli, Technol. Forecast. Soc. Change 74, 247 (2007).

[38] K. Namwoon, E. Bridges, R.K. Srivastava, Int. J. Res. Market. 16, 95 (1999). 